\title{
Generation of incoherent Cherenkov diffraction radiation in synchrotrons
}

\author{
R. Kiefferø, ${ }^{1}$ L. Bartnik, ${ }^{4}$ M. Bergamaschi $\odot,{ }^{1}$ V. V. Bleko $\odot,{ }^{2}$ M. Billing, ${ }^{4}$ L. Bobb $\odot,{ }^{5}$ \\ J. Conway $\odot,{ }^{4}$ M. Forster, ${ }^{4}$ P. Karataev, ${ }^{6}$ A. S. Konkov $\odot,{ }^{2}$ R. O. Jones $\odot,{ }^{1}$ T. Lefevre $\odot,{ }^{1}$ \\ J. S. Markova, ${ }^{3}$ S. Mazzoni $\odot,{ }^{1}$ Y. Padilla Fuentes $\odot,{ }^{4}$ A. P. Potylitsyn, ${ }^{3}$ J. Shanks $\odot,{ }^{4}$ and S. Wang ${ }^{4}$ \\ ${ }^{1}$ CERN, Geneva, 1211, Switzerland \\ ${ }^{2}$ Joint Institute for Nuclear Research, Dubna, 141980 Russia \\ ${ }^{3}$ Tomsk Polytechnic University, Tomsk, 634050 Russia \\ ${ }^{4}$ Cornell University, Ithaca, New York, 14850 USA \\ ${ }^{5}$ Diamond Light Source, Oxfordshire, OX11 ODE, United Kingdom \\ ${ }^{6}$ Royal Holloway, University of London, Egham, TW20 OEX, United Kingdom
}

(Received 15 January 2020; accepted 6 April 2020; published 24 April 2020)

\begin{abstract}
Incoherent Cherenkov diffraction radiation was recently produced in the Cornell electron storage ring using counterpropagating beams (electrons and positrons) passing in the close vicinity of a dielectric made of fused silica. We present in this paper a collection of the experimental investigations that were performed on Cherenkov diffraction radiation in both the infrared and the visible range. Measurements were performed using an optical system functioning either in imaging conditions or in far field conditions to retrieve the angular distribution of the radiation. Polarization studies were also performed and showed that, when selecting the appropriate polarization, the beam size can be measured accurately. This study opens the path for new applications in noninvasive beam diagnostic for highly relativistic charged particle beams.
\end{abstract}

DOI: 10.1103/PhysRevAccelBeams.23.042803

\section{INTRODUCTION}

Since its discovery in the mid 1930s, the usage of Cherenkov radiation (ChR) [1] has widely spread as a technique to detect charged particles in many different fields such as nuclear [2] and particle [3] physics or astrophysics [4]. Recently, a first experiment was performed to investigate the possibility of noninvasive beam diagnostic techniques based on the detection of incoherent Cherenkov diffraction radiation (ChDR) [5]. The latter refers to the emission of Cherenkov radiation by charged particles traveling not inside, but in the vicinity of, a dielectric material. This combines the already well-known advantages of Cherenkov radiation with noninvasive photon generation, making it an ideal technique for beam instrumentation. Those investigations were performed at the Cornell Electron Storage Ring (CESR) using positrons passing in the close vicinity of a 2 -cm-long radiator made out of fused silica. In this paper, we present a full collection of all the experimental investigations that were performed at CESR to study the properties of $\mathrm{ChDR}$ and investigate its potential for beam diagnostics in circular accelerators. The

Published by the American Physical Society under the terms of the Creative Commons Attribution 4.0 International license. Further distribution of this work must maintain attribution to the author(s) and the published article's title, journal citation, and DOI. tests were performed using both positrons and electrons at 2.1 or $5.3 \mathrm{GeV}$.

\section{CHERENKOV DIFFRACTION RADIATION}

Cherenkov diffraction radiation can be considered as polarization radiation $(\mathrm{PR})[6,7]$ resulting from polarization currents in the volume of a dielectric induced by the electromagnetic field of a passing particle. In a simple prismatic geometry, such as the one presented in Fig. 1, a charged particle, propagating at a distance $b$ from the surface of the dielectric, would emit photons at the Cherenkov angle, i.e., $\theta_{C h R}=\cos ^{-1}(1 / \beta n)$ with $\beta$ being the speed of the particle normalized to the speed of light in a vacuum and $n$ being the index of refraction of the dielectric. The photons are emitted along the length of the prism, denoted $a$, and refracted out as they reach the exit surface of the prism, that is tilted by an angle $\varphi$. The angle of emission of diffraction radiation (DR) emitted at the entrance surface of the prism is also shown in Fig. 1. It will be internally reflected and would leave the prism at a different angle than the Cherenkov radiation such that DR will not be measured.

The corresponding spectral-angular distribution of Cherenkov diffraction radiation as defined in Ref. [7] can be written for the polarizations being parallel and perpendicular to the beam propagation as per Eqs. (1) and (2): 


$$
\begin{aligned}
\frac{d^{2} W_{\|}}{d \lambda d \Omega}= & D \cdot\left|\frac{\varepsilon(\lambda)}{\varepsilon(\lambda) \cos (\theta-\delta)+U}\right|^{2} \mid \cos (\delta)\left[\gamma^{-1} \sin (\theta-\delta)-i K U \cos (\phi)\right]+\sin (\delta)\left[i K \sin (\theta-\delta)+\gamma^{-1} U \cos (\phi)\right] \\
& -\left.\gamma \beta U \sin (\theta-\delta) \sin ^{2}(\phi)\right|^{2},
\end{aligned}
$$

$$
\begin{aligned}
\frac{d^{2} W_{\perp}}{d \lambda d \Omega}= & D \cdot \gamma^{2} \sin ^{2}(\phi)\left|\frac{\sqrt{\varepsilon(\lambda)}}{\cos (\theta-\delta)+U}\right|^{2}\left[\sin ^{2}(\theta-\delta)+|U|^{2}\right] \cdot\left[1-\beta^{2} \cos ^{2}(\theta-\delta)+2 \beta \gamma^{-2} \sin (\delta) \sin (\theta-\delta) \cos (\phi)\right. \\
& \left.-\gamma^{-2} \sin ^{2}(\delta)\left(K^{2}-\gamma^{-2}\right)\right]^{2}
\end{aligned}
$$

with

$$
\begin{aligned}
D= & \frac{\alpha}{2 \pi^{2}} \frac{\hbar c}{\lambda^{2}} \frac{\beta^{2} \cos ^{2}(\theta-\delta)}{K^{2}|P|^{2}}\left|\frac{\varepsilon(\lambda)-1}{\varepsilon(\lambda)}\right|^{2}\left|1-\exp \left[-i a \frac{2 \pi}{\beta \lambda}[P+\Sigma \cdot \cot (\varphi)] \sin (\varphi)\right]\right|^{2} \\
& \times \frac{\exp \left[-2 \frac{2 \pi}{\gamma \beta \lambda}[h+a \cdot \cos (\varphi) K \cos (\delta)]\right]}{1-\beta^{2} \cos ^{2}(\theta-\delta)+\beta^{2} \sin ^{2}(\delta)\left[1-\sin ^{2}(\theta-\delta) \sin ^{2}(\phi)\right]+2 \beta \sin (\delta) \sin (\theta-\delta) \cos (\phi)}, \\
U= & \sqrt{\epsilon(\lambda)-\sin ^{2}(\theta-\delta),} \quad K=\sqrt{1+[\gamma \beta \sin (\theta-\delta) \sin (\phi)]^{2}}, \quad P=\cos (\delta)-\beta U+i \gamma^{-1} K \sin (\delta), \\
\Sigma= & \sin (\delta)+\beta \sin (\theta-\delta) \cos (\phi)-i \gamma^{-1} K \cos (\delta), \quad K=\sqrt{1+[\gamma \beta \sin (\theta-\delta) \sin (\phi)]^{2}},
\end{aligned}
$$

where $\varepsilon$ is the dielectric permittivity of the target material, $\omega$ is the frequency of the emitted radiation, $\theta$ and $\phi$ are the polar and the azimuthal observation angles, respectively (measured from the particle direction of motion), and $\delta$ is the angle of flight of the charged particle relative to the $z$ axis. Using the Cherenkov and Snell laws, $\theta$ can be obtained as

$$
\theta=\cos ^{-1}\left(\frac{\cos \varphi}{\beta}-\sin \varphi \sqrt{n^{2}-\frac{1}{\beta^{2}}}\right)-\varphi
$$

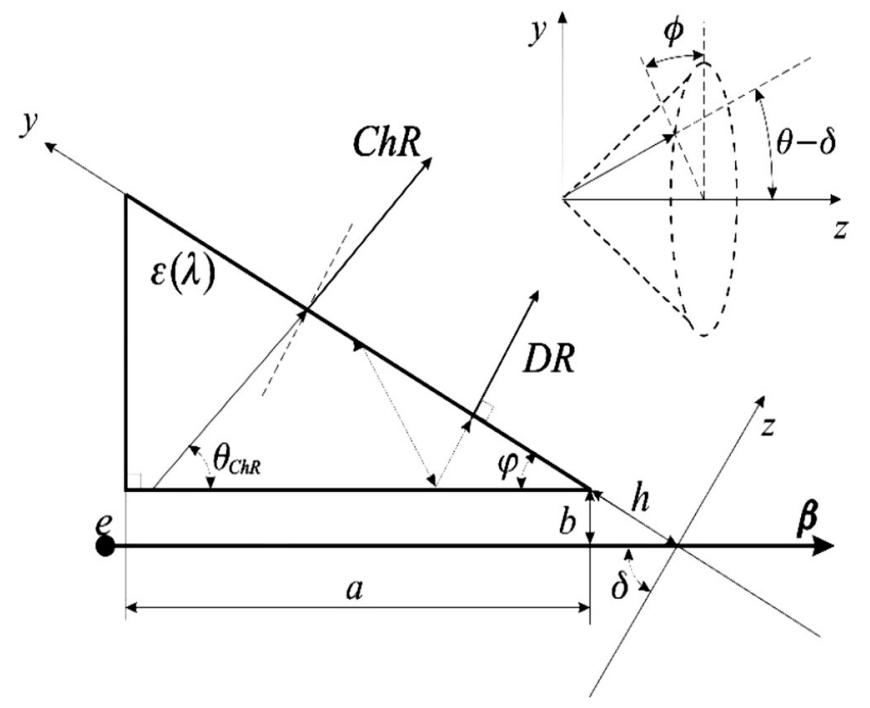

FIG. 1. Emission of Cherenkov diffraction radiation by a charged particle propagating at a distance $b$ from the surface of a dielectric material.
An example of the angular spectral density emitted by a $5.3 \mathrm{GeV}$ electron propagating at several distances from the surface of a 2-cm-long dielectric made out of fused silica is given in Fig. 2, showing both the horizontal and vertical polarization components of the radiation. In these simulations, the angle $\varphi$ of the prism is $29^{\circ}$.

In contrast with the emission of conventional Cherenkov radiation, where polarization is strictly radial, in ChDR one can detect only a fraction of the Cherenkov radiation cone. Because of such azimuthal symmetry violation, ChDR has both polarization components. Here the vertical polarization coincides with the radial direction and lies in the plane containing the particle momentum and a wave vector. The other polarization component, i.e., horizontal, is a tangential one and is typically absent in conventional Cherenkov radiation. In the prismatic geometry we considered, both polarizations are emitted in a narrow cone centered on the Cherenkov angle $\left(\theta_{\mathrm{ChR}} \cong 46.4^{\circ}\right)$, which is then refracted out at an angle $\theta \cong 40^{\circ}$. The expected single-particle azimuthal $(\phi)$ and polar $(\theta)$ angular distributions of both horizontally and vertically polarized photons are shown in Fig. 2 for different impact parameters. With an increasing impact parameter, the radiation intensity decreases significantly and the angular distributions get slightly narrower. For $\phi=0^{\circ}$ and $\theta=\theta_{\mathrm{ChR}}$, due to symmetry, the azimuthal distribution of horizontally polarized photons is null. For nonzero angle $\phi$, the symmetry is broken, and the horizontal component is appearing.

The photon spectrum emitted by a single particle has also been calculated, and the results are presented in Fig. 3 for different impact parameters and beam energies of 2.1 and $5.3 \mathrm{GeV}$. The radiation power increases with higher beam energies. For realistic impact parameters ranging from 1 to 

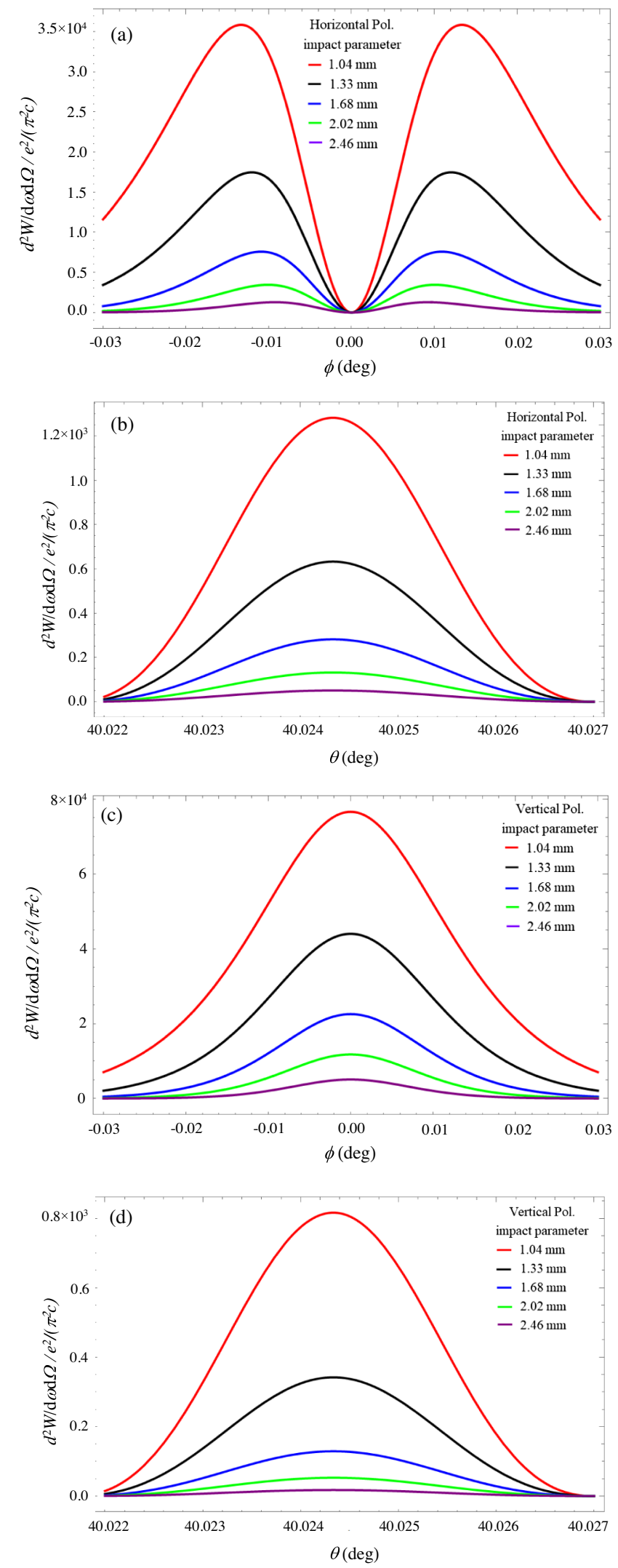

FIG. 2. Angular distributions simulated for $5.3 \mathrm{GeV}$ positrons using visible radiation. Azimuthal (a) and polar (b) distributions of horizontally polarized photons and the azimuthal (c) and polar (d) distributions of vertically polarized photons.

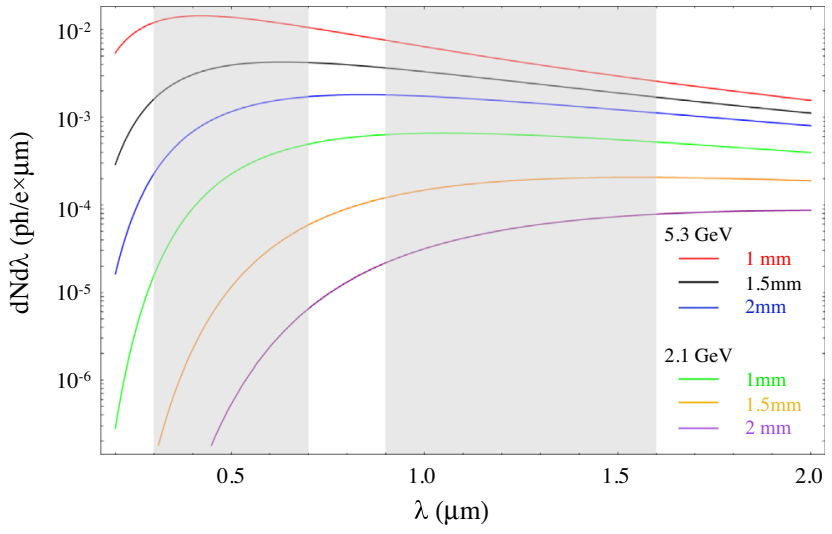

FIG. 3. Cherenkov diffraction photon spectrum calculated for 2.1 and $5.3 \mathrm{GeV}$ electrons or positrons and impacts parameters of $1,1.5$, and $2 \mathrm{~mm}$.

$2 \mathrm{~mm}$, the radiation spectrum peaks in the near infrared and in the visible range for 2.1 and $5.3 \mathrm{GeV}$ electron beam energies, respectively. In the model, the dielectric properties of the target are defined from the three-term Sellmeier dispersion equation [8]. In Fig. 3, the intensity suppression at shorter wavelengths appears due to the finite impact parameter. On the other hand, at longer wavelengths the suppression is caused by the diffraction effects due to finite dimensions of the target.

\section{EXPERIMENTAL SETUP}

\section{A. Cornell Electron-Positron Storage Ring}

The Cornell Electron Storage Ring was designed and operated as an electron and positron collider since 1979. In the early 2000s, the high-energy physics program on CESR stopped, but the accelerator was kept operational as a test facility to study beam dynamics and instrumentation [9] and alternatively as a synchrotron light source. The experimental chamber is located in one of the straight sections of the storage ring. This location was chosen to reduce the synchrotron radiation background from bending magnets upstream. Experiments were performed using a single bunch with either electrons or positrons and even both at the same time. Two testing campaigns have been organized separately, running the synchrotron at two different beam energies, i.e., 2.1 or $5.3 \mathrm{GeV}$. The beam parameters of CESR are presented in Table I.

\section{B. Vacuum vessel}

The experimental setup uses a vacuum tank, depicted in Fig. 4, initially built and installed in 2010 for noninvasive beam size measurements using diffraction radiation from dielectric slits [10]. The ChDR radiator, described in more detail in the next paragraph, is mounted on a mechanism with two degrees of freedom: translation to insert the radiator once the beam is circulating in the storage ring 
TABLE I. Parameters of CESR.

\begin{tabular}{lc}
\hline \hline Ring circumference & $768.4 \mathrm{~m}$ \\
Revolution time & $2.563 \mu \mathrm{s}$ \\
Beam energy & 2.1 and $5.3 \mathrm{GeV}$ \\
Beam species & $e^{-}$and $e^{+}$ \\
Particles per bunch & $1.6 \times 10^{10}$ \\
\hline \hline
\end{tabular}

and rotation to allow precise steering of the emitted photons through the optical detection line.

\section{The radiator}

The radiator, made of high-purity fused silica, $\mathrm{SiO}_{2}$ (7980 from Corning), has a prismatic shape as depicted in Fig. 5. With an index of refraction of 1.46, the particles would emit $\mathrm{ChDR}$ photons at an angle of $46.4^{\circ}$ with respect to the beam trajectory. As we mentioned in the introduction, this target was conceived to measure ChDR from counterpropagating beams. As the positrons travel from the left to right in Fig. 5, they emit ChDR along the length of the radiator. When the photons reach the outer face of the radiator (inclined at $29^{\circ}$ with respect to the beam trajectory), they are refracted toward the detection system, which was designed to be centered at an angle of $40^{\circ}$ relative to the incoming beam trajectory. The electrons, coming from the opposite direction, will produce ChDR as represented in blue in Fig. 5. These photons are reflected inside the radiator toward the detection system by a highly reflective aluminium layer coating the $90^{\circ}$ face of the radiator. The radiation emitted by electrons and positrons is thus refracted out of the radiator at the same angle but would exit the radiator at different longitudinal positions as shown in Fig. 5 in blue for electrons and red for positrons. The images of the electron and positron beams would therefore appear on different locations of the radiator and could be distinguished in this respect.

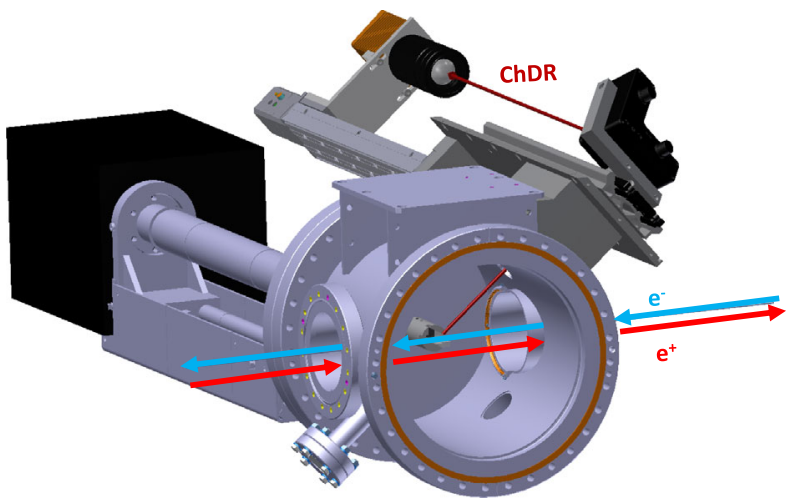

FIG. 4. The 3D layout of the vacuum chamber including the radiator and the optical detection system.

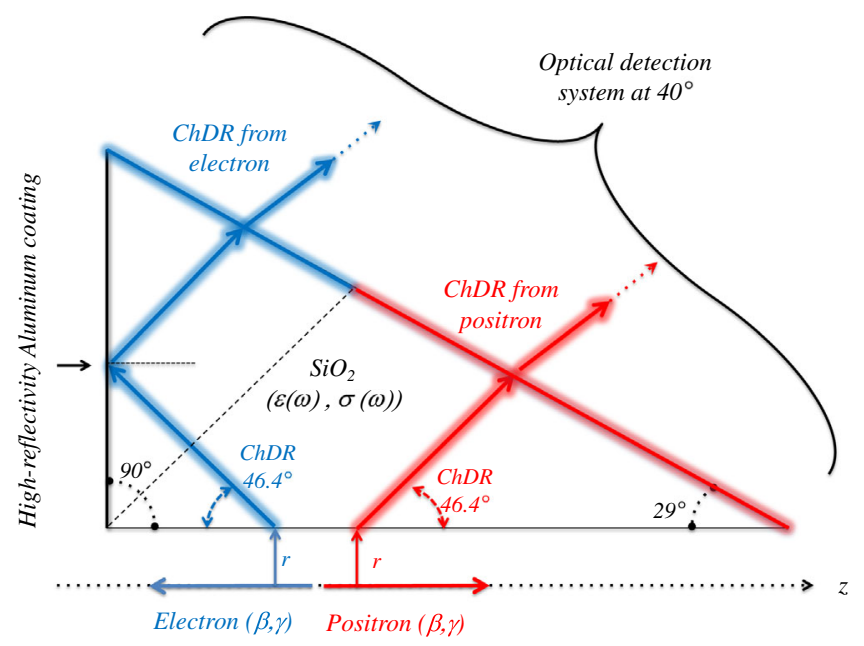

FIG. 5. Sketch of a prismatic target for the observation of counterpropagating beams. Positrons are going left to right and electrons right to left.

\section{Detection systems}

Between the different testing campaigns on CESR, the detection system has been optimized using cameras and optical components adapted to the infrared and the visible range when running with 2.1 and $5.3 \mathrm{GeV}$ beam energies, respectively.

In the infrared, the detection system is based on an InGaAs camera [11] equipped with a sensor of $640 \times$ 512 pixels with a pitch of $20 \mu \mathrm{m}$. Sensitive to the wavelength range from $800 \mathrm{~nm}$ to $1.7 \mu \mathrm{m}$, the camera reaches a quantum efficiency up to $80 \%$ for a $1.5 \mu \mathrm{m}$ wavelength. The camera has an integration time window adjustable from $1 \mu \mathrm{s}$ to $40 \mathrm{~ms}$ and acquires images with a 14 bits dynamic range. The camera is equipped with a $50 \mathrm{~mm}$ focal length, $f / 2.15$ numerical aperture objective lens, providing a magnification of $1 / 15$ with a depth of field of $4 \mathrm{~cm}$.

In the visible range, the photons are detected by an intensified camera equipped with a multialkali photocathode sensitive to wavelengths between 300 and $700 \mathrm{~nm}$. Two lenses, mounted on flippers, can be inserted either to image the radiator using a $150 \mathrm{~mm}$ focal length achromatic lens or to measure the angular distribution of the radiation with a $500 \mathrm{~mm}$ focal length plano-convex lens. Experimentally, the best signal to noise ratio on the camera is achieved by adjusting both the gain of the image intensifier and the integration time of the camera. With more photons being produced at $5.3 \mathrm{GeV}$, as shown in Fig. 3, the optical system was also equipped with a polarizer mounted on a remotely controlled rotation stage and a set of $10 \mathrm{~nm}$ bandpass optical filters at 400 and $600 \mathrm{~nm}$.

\section{EXPERIMENTAL RESULTS}

\section{A. High directivity of the emission}

Images acquired for electrons and positrons at $5.3 \mathrm{GeV}$ are depicted in Fig. 6, superimposed on the 3D model of the 


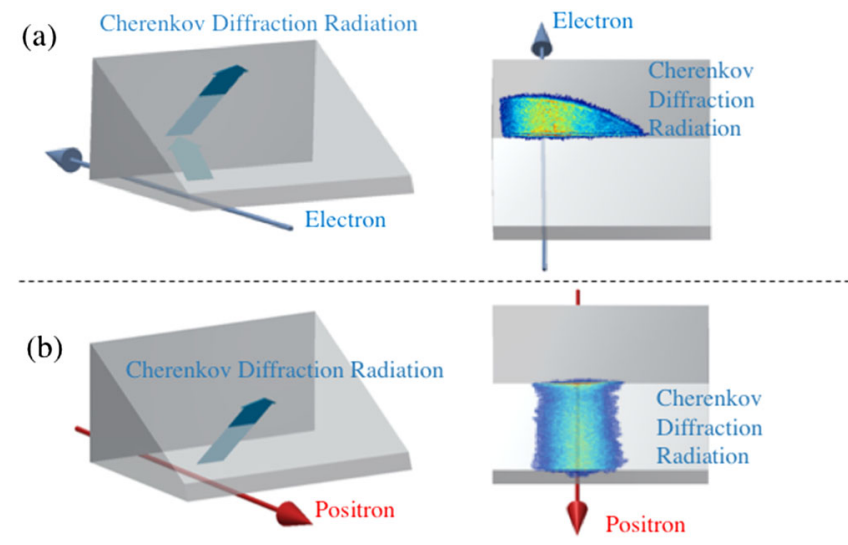

FIG. 6. Images inserted in a pictorial layout in order to clarify where and how light is produced inside the radiator: measurements performed in the visible range for $5.3 \mathrm{GeV}$ electrons (a) and positrons (b).

radiator. The images from electrons and positrons appear in the different zones as described in Fig. 5, which validate the emission angle of the light and the strong directivity of this radiation as expected.

The angular acceptance of the optical system is limited by the physical aperture of the viewport and of the first mirror in the line, as it was originally built to image a $1 \mathrm{~mm}$ large object. With the present system, it is then impossible to image properly a 2-cm-long target. In Fig. 6, the measurements presented were optimized for imaging positrons. In this condition, the image of the electron beam is strongly clipped by a circular aperture. The optical components of the detection line could, however, be realigned to provide a better image of the electron beam as shown later in this paper.

\section{B. Angular distributions}

The angular distribution of the ChDR was studied in detail experimentally at a wavelength of $600 \mathrm{~nm}$ using positrons at $5.3 \mathrm{GeV}$. As the radiation is confined in a submilliradian cone angle, the measurements are typically performed using a low gain on the image intensifier and a $1.2 \mathrm{~ms}$ camera integration time. To illustrate the nonisotropic polarization of the ChDR field, a series of images have been acquired for different polarization states and are shown in Fig. 7. As the radiator is located above the beam, our model also predicts a stronger emission of vertically polarized photons that is also measured experimentally as depicted in Fig. 7. Using such a prismatic radiator, vertically polarized photons are directly refracted toward the camera, contrarily to other polarization states that would have slightly different output angles as they exit the radiator. Those photons would thus spread out in the horizontal plane. The calculated single-particle angular distribution for horizontal polarization, presented in Fig. 2, has a minimum for $\phi=0^{\circ}$. Experimentally, this is not observed, as we need to consider the horizontal beam divergence of $0.27 \mathrm{mrad}$ as measured on CESR [12]. In this case, we obtain a significantly broader distribution with a maximum for $\phi=0^{\circ}$, compatible with the measurements presented in Fig. 7.

The angular distributions of vertically polarized photons have been also studied as a function of the impact parameter. The results are presented in Fig. 8, and the width of the distributions is compared to our theoretical predictions in Table II. The theoretical model uses singleparticle distributions as shown in Fig. 2 and then convolutes them with the horizontal and vertical beam divergences of 270 and $18 \mu \mathrm{rad}$, respectively, as extrapolated from emittances measured using synchrotron light monitors [12]. The agreement between calculations and experiments, presented in Table II, is very good and also confirms a decrease of the angular distributions for larger impact parameters.

\section{Beam imaging using ChDR}

In imaging conditions, measurements were performed using either electrons or positrons and at different beam energies. As examples, we present a series of images acquired for different impact parameters using electrons at $2.1 \mathrm{GeV}$ (Fig. 9) and positrons at $5.3 \mathrm{GeV}$ (Figs. 10 and 11). One should note that those measurements were performed using different cameras and optical components as was previously mentioned. Both images and horizontal beam profiles are depicted in these figures. The profiles are obtained by integrating the pixels in the image over a
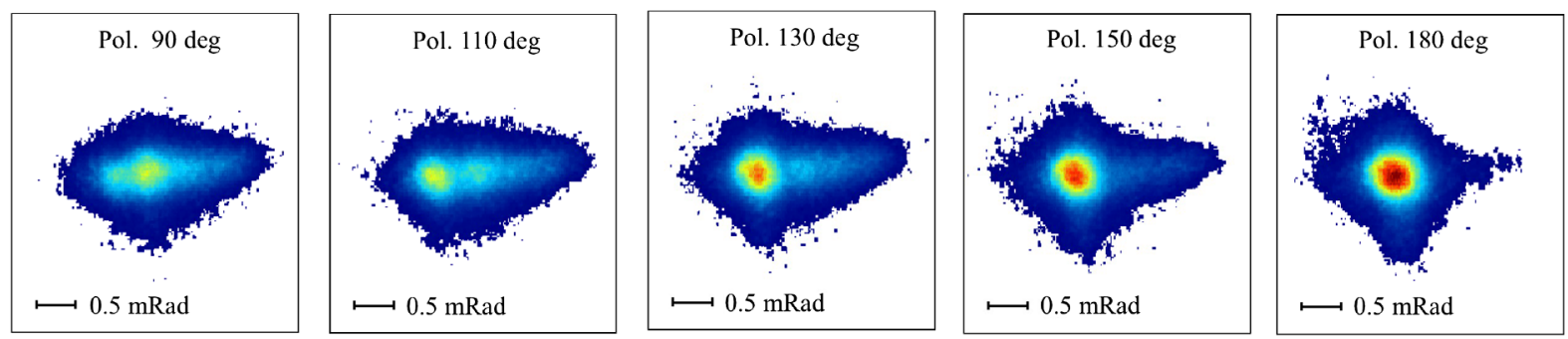

FIG. 7. Angular distributions of Cherenkov diffraction radiation for different polarizations measured at $600 \pm 10 \mathrm{~nm}$ for $5.3 \mathrm{GeV}$ positrons. $90^{\circ}$ is horizontal polarization and $180^{\circ}$ vertical polarization. 
TABLE II. Comparison between the calculated and measured widths of vertically polarized angular distributions for different impact parameters.

\begin{tabular}{llllll}
\hline \hline Impact parameters $(\mathrm{mm})$ & 1.04 & 1.33 & 1.68 & 2.02 & 2.46 \\
\hline FWHM $_{\text {exp }}(\mathrm{mrad})$ & 0.54 & 0.49 & 0.47 & 0.45 & 0.43 \\
FWHM $_{\text {the }}(\mathrm{mrad})$ & 0.56 & 0.49 & 0.47 & 0.44 & 0.40 \\
\hline \hline
\end{tabular}

distance of $2 \mathrm{~mm}$ along the beam path. One may see that the intensity reduces exponentially when the impact parameter increases. This peculiarity is well described in Sec. IV D.

From our theoretical model [7], we expect that the current radiator geometry would provide the possibility to measure the horizontal beam profile using vertically polarized photons. A series of beam images and the corresponding horizontal profiles have been acquired for different polarization states. The results are presented on Figs. 10 and 11 for vertical and horizontal polarizations, respectively. It shows that horizontal beam profiles from horizontally polarized light are distorted with the appearance of asymmetric tails. The same measurements performed using vertically polarized photons provide undistorted profiles which are in good agreement with the horizontal beam size measured using synchrotron radiation monitors [12].

It is also observed in Figs. 9-11 that the measured beam size increases slightly for increasing impact parameters.
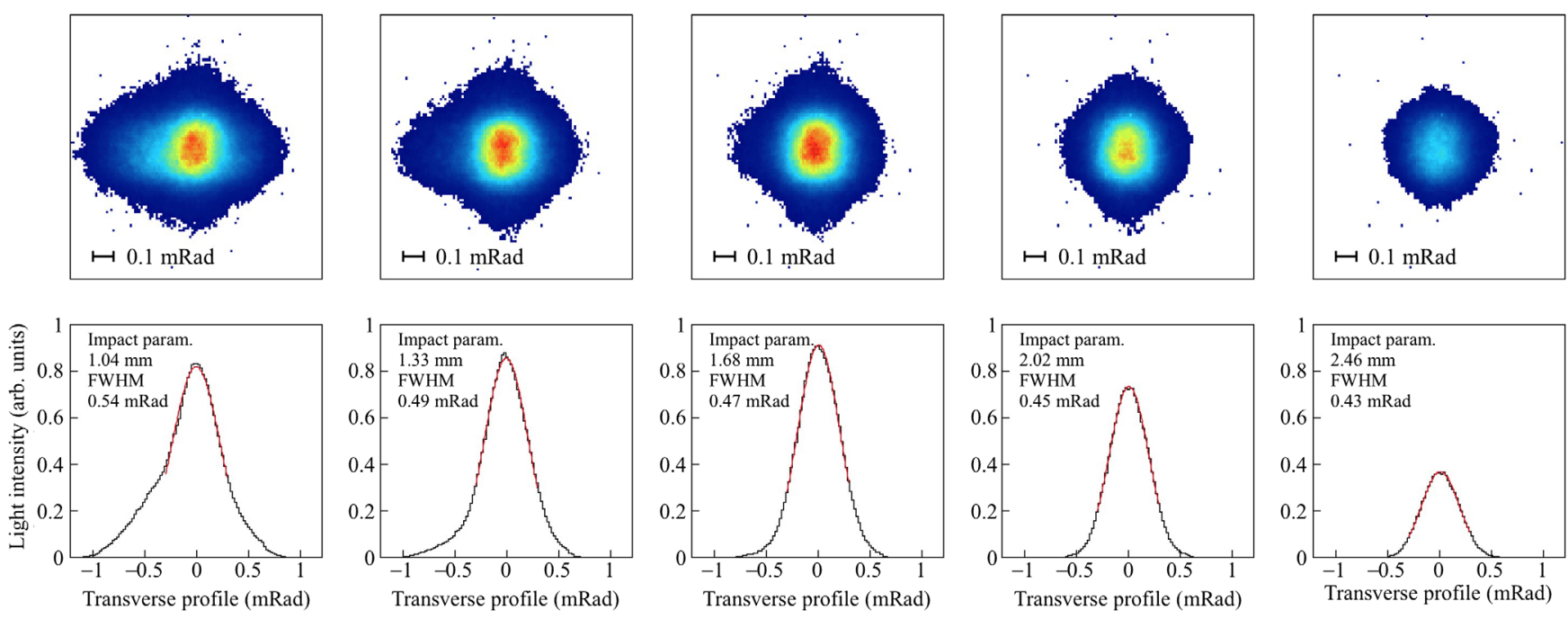

FIG. 8. Angular distributions of vertically polarized photons measured with $5.3 \mathrm{GeV}$ positrons using visible radiation as a function of the impact parameter. Images (top) and polar angle distributions (bottom).
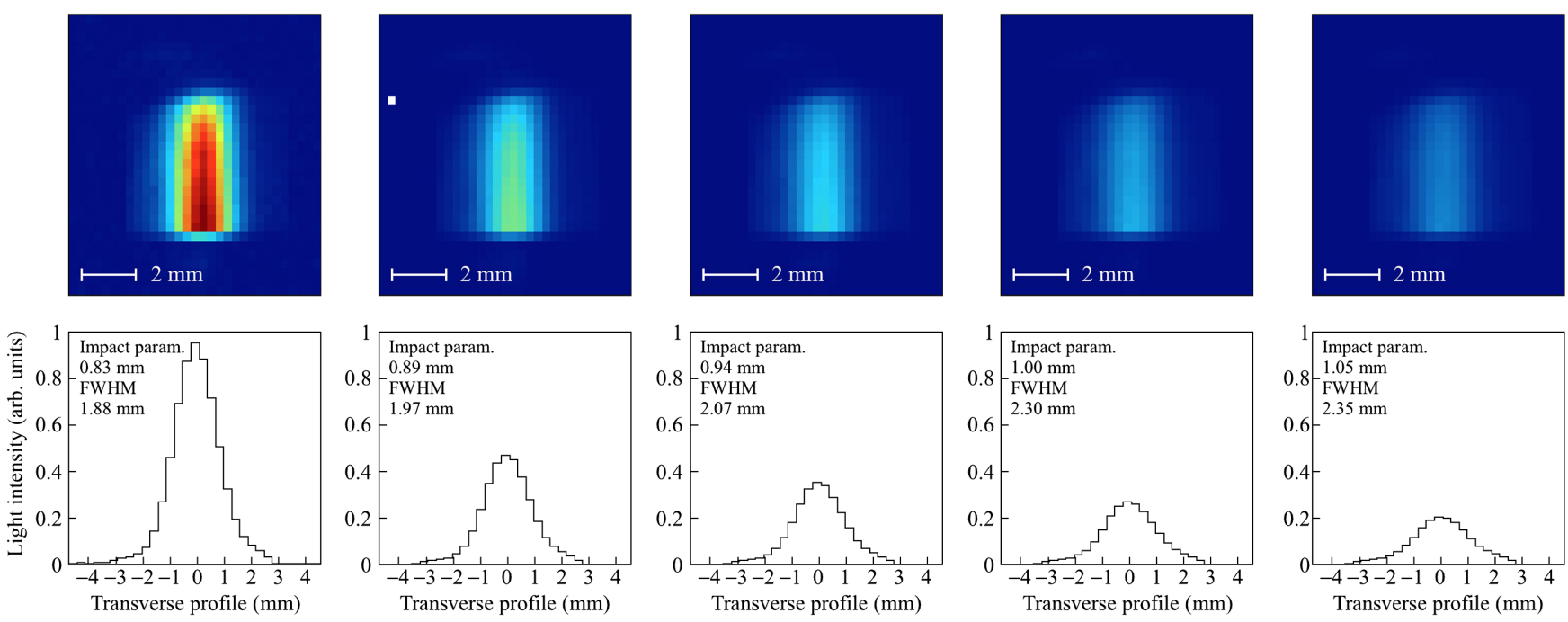

FIG. 9. Image and profiles taken with $2.1 \mathrm{GeV}$ electrons using infrared radiation with no polarizer and no bandpass filter. Image (top) and horizontal profile (bottom). 

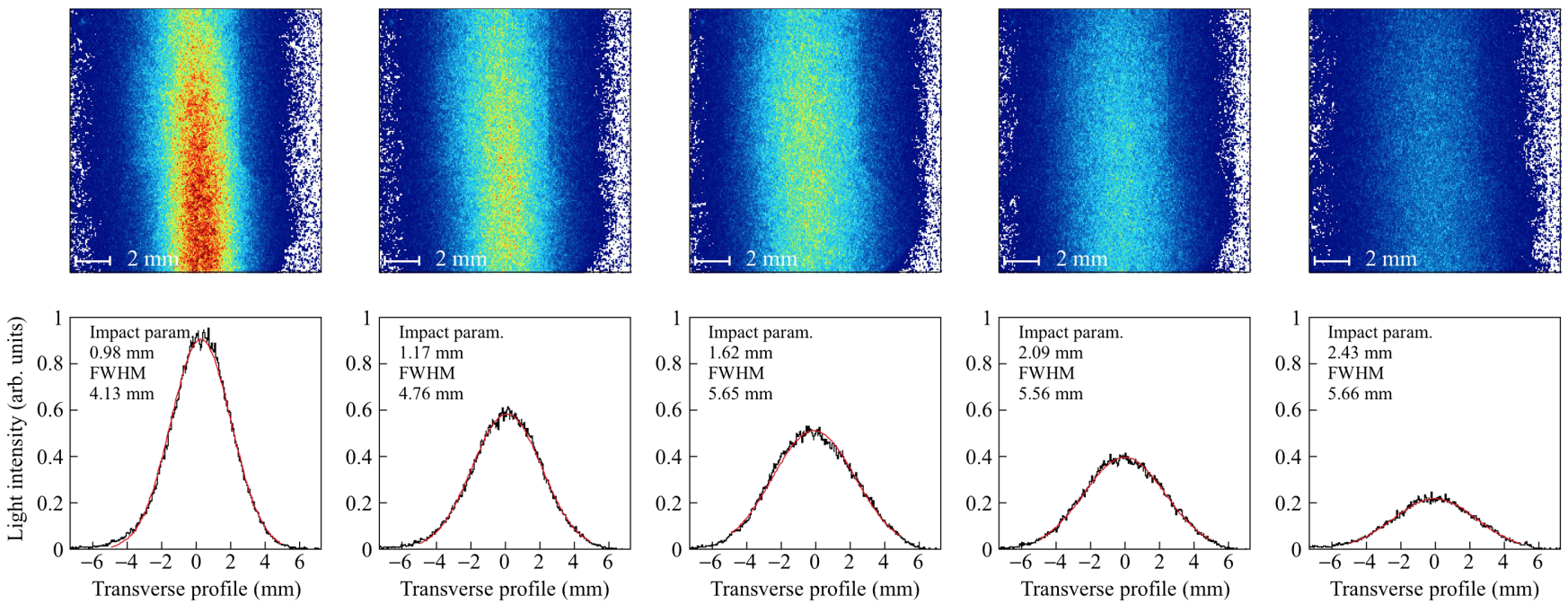

FIG. 10. Imaging positrons of $5.3 \mathrm{GeV}$ at $600 \pm 10 \mathrm{~nm}$ and vertical polarizer. Image (top) and horizontal profile (bottom).
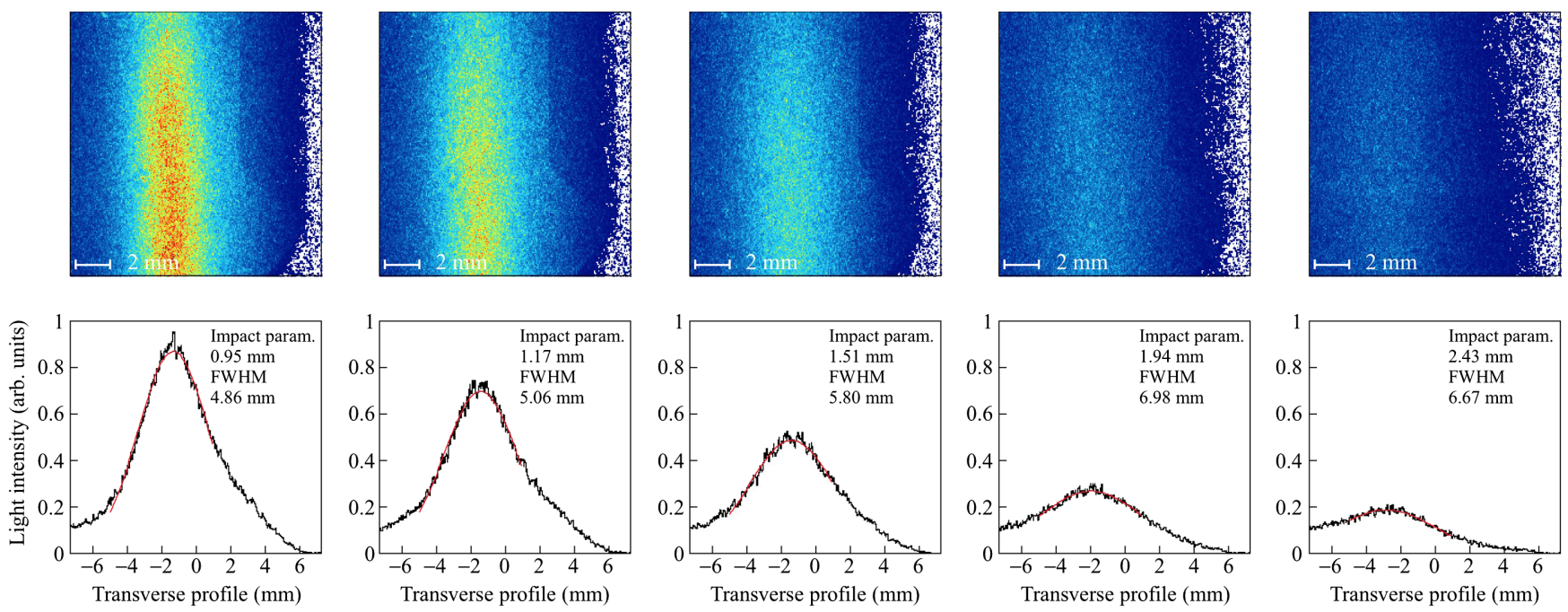

FIG. 11. Imaging positrons of $5.3 \mathrm{GeV}$ at $600 \pm 10 \mathrm{~nm}$ and horizontal polarizer. Image (top) and horizontal profile (bottom). On the other hand, the intensity along the particle trajectory is not uniform, either. It suggests that the beam trajectory is not parallel to the interface. Therefore, the orientation of the ChDR and intensity distribution along the beam trajectory provide an opportunity to monitor the beam trajectory in two dimensions simultaneously.

The measured horizontal beam size evolution is plotted in Fig. 12 as a function of the impact parameter. The measurements are taken for $5.3 \mathrm{GeV}$ electrons, using a $600 \pm 10 \mathrm{~nm}$ wavelength filter and vertically polarized photons. An increase in the beam size from 1.7 to $2.5 \mathrm{~mm}$ is measured for impact parameters ranging from 0.6 to $2.3 \mathrm{~mm}$. This would suggest that the line spread function of ChDR is increasing with impact parameters. Our theoretical model is currently providing only the angular spectral characteristics of the radiation produced. Modifications to this model have been initiated in order to estimate the ChDR line spread function that would give the image produced by a single particle.
In order to have an estimation of the spatial resolution of the system, we have been recording the variation of the centroid of measured beam profile acquired in 100 consecutives images. The results are presented in Fig. 13, showing a variance of $13.31 \mu \mathrm{m}$ in the horizontal beam position measured, which is compatible with the current beam stability measured on CESR by beam position monitors.

\section{Photon yield as a function of the impact parameter}

By integrating over all the pixels, we calculate the relative increase in light yield as a function of the impact parameters. The results are presented for electrons of 2.1 


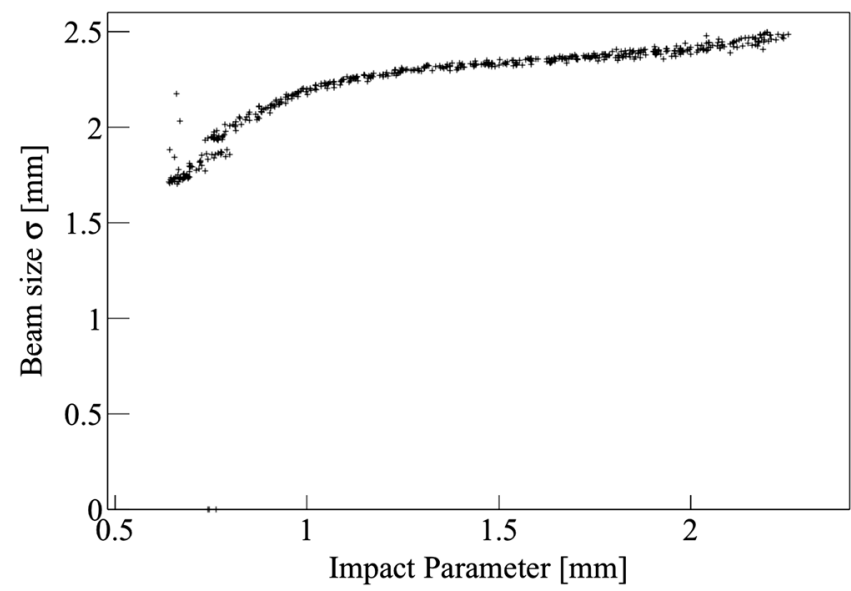

FIG. 12. Measured beam size as a function of the impact parameter using vertically polarized photons at $600 \pm 10 \mathrm{~nm}$ for $5.3 \mathrm{GeV}$ electrons.

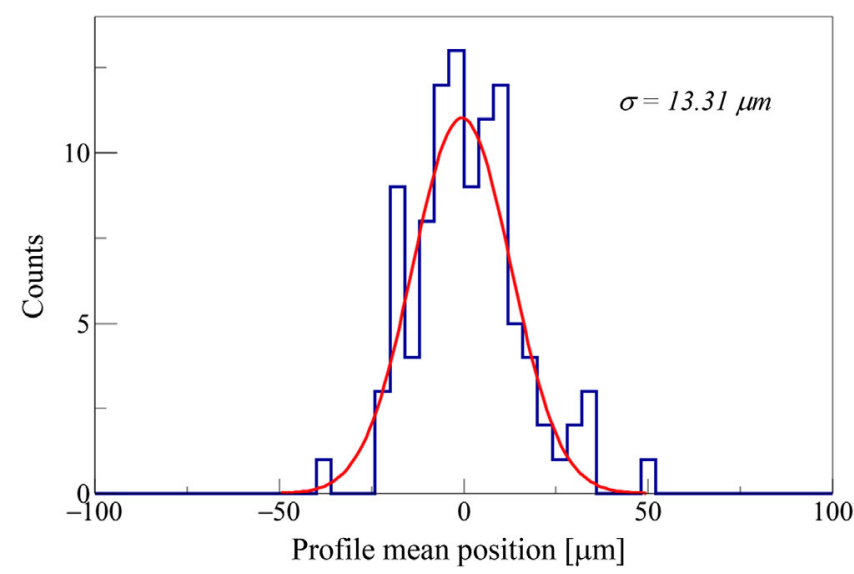

FIG. 13. Stability of the measured beam centroid position over 100 consecutive images acquisitions using vertically polarized photons at $600 \pm 10 \mathrm{~nm}$ for $5.3 \mathrm{GeV}$ positrons.

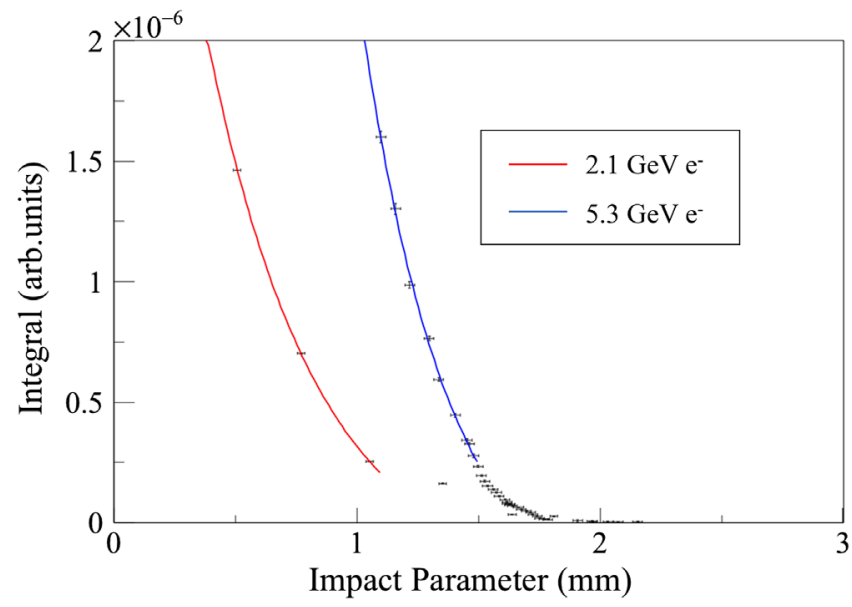

FIG. 14. Measured relative light intensity as a function of the impact parameters emitted by electrons of $2.1 \mathrm{GeV}$ in the NIR using no filter and by electrons of $5.3 \mathrm{GeV}$ at a wavelength of $600 \pm 10 \mathrm{~nm}$ using a vertical polarizer. and $5.3 \mathrm{GeV}$ in Fig. 14. As expected from the theoretical photon spectrum presented in Fig. 3, the light intensity is exponentially decreasing for larger impact parameters. The data presented in Fig. 14 have been calibrated to our best knowledge considering the sensitivity of the near infrared and visible cameras and the transmission losses in the corresponding optical systems.

We also measured that the light intensity produced by positrons at $5.3 \mathrm{GeV}$ is twice smaller for a wavelength of $400 \pm 10 \mathrm{~nm}$ than for $600 \pm 10 \mathrm{~nm}$, which is in good agreement with our theoretical prediction (see Fig. 3).

\section{CONCLUSION}

We presented the results of an extensive study on incoherent Cherenkov diffraction radiation produced by 2.1 and $5.3 \mathrm{GeV}$ electrons and positrons circulating on the Cornell storage ring. The angular and spectral properties of the radiation have been investigated in both the visible and the near infrared range and were found in good agreement with our theoretical model. The directivity of the radiation was found good enough to provide fully independent measurements of counterpropagating beams using the same setup. The intensity distribution along the beam direction enables us to perform a simultaneous two-dimensional trajectory monitoring. Beam imaging using ChDR has also been studied, showing that, in our configuration, vertically polarized photons are providing good measurements of the horizontal beam profile of the beam for smaller impact parameters, i.e., around $1 \mathrm{~mm}$. It was also observed that the measured beam size increases with impact parameters. This will actually be studied analytically in future work. In addition, with horizontal beam sizes of a few millimeters, the line spread function of ChDR could not be studied experimentally on CESR, and we cannot estimate accurately the resolution limit of such a technique. To address this point, further studies are actually planned on the Advanced Test Facility 2 (ATF2) at KEK, where very small beam sizes, i.e., a few microns, can be obtained and measured accurately $[13,14]$.

ChDR diagnostics could be applied to a wide range of accelerators, from high-energy lepton and hadron colliders to synchrotron light sources and free electron laser facilities. They should also be capable to provide very compact optically based monitors, that in the framework of novel accelerating technologies could be embedded in very small dielectric tubes (i.e., $<2 \mathrm{~mm}$ aperture), providing reliable measurements of both the beam position and profile [15].

\section{ACKNOWLEDGMENTS}

The authors thank the Ministry of Education and Science of the Russian Federation for its Grant No. FSWW2020-0008. 
[1] P. A. Cherenkov, Visible radiation produced by electrons moving in a medium with velocities exceeding that of light, Phys. Rev. 52, 378 (1937).

[2] M. Demarteau, R. Lipton, H. Nicholson, and I. Shipsey, Particle and nuclear physics instrumentation and its broad connections, Rev. Mod. Phys. 88, 045007 (2016).

[3] C. W. Fabjan and F. Gianotti, Calorimetry for particle physics, Rev. Mod. Phys. 75, 1243 (2003).

[4] T. Kajita, Nobel lecture: Discovery of atmospheric neutrino oscillations, Rev. Mod. Phys. 88, 030501 (2016).

[5] R. Kieffer et al., Direct Observation of Incoherent Cherenkov Diffraction Radiation in the Visible Range, Phys. Rev. Lett. 121, 054802 (2018).

[6] D. V. Karlovets and A. P. Potylitsyn, Diffraction radiation from a finite-conductivity screen, J. Exp. Theor. Phys. 90, 326 (2009).

[7] M. V. Shevelev and A.S. Konkov, Peculiarities of the generation of Vavilov-Cherenkov radiation induced by a charged particle moving past a dielectric target, J. Exp. Theor. Phys. 118, 501 (2014).

[8] E. D. Palik, Handbook of Optical Constants of Solids (Academic, San Diego, 1998).

[9] M. Billing et al., The conversion of CESR to operate as the Test Accelerator, CesrTA. Part 1: Overview, J. Instrum. 10, P07012 (2015).
[10] L. Bobb, R. Kieffer, T. Lefevre, S. Mazzoni, T. Aumeyr, P. Karataev, M. Billing, J. Conway, and J. Shanks, Feasibility of diffraction radiation for non- invasive beam diagnostics as characterized in a storage ring, Phys. Rev. Accel. Beams 21, 032801 (2018).

[11] Model Bobcat from Xenics infrared solution: http://www .xenics.com/en/bobcat-640-gige.

[12] S. T. Wang, D. L. Rubin, J. Conway, M. Palmer, D. Hartill, R. Campbell, and R. Holtzapple, Visible-light beam size monitors using synchrotron radiation at CESR, Nucl. Instrum. Methods Phys. Res., Sect. A 703, 80 (2013).

[13] P. Karataev, S. Araki, R. Hamatsu, H. Hayano, T. Muto, G. Naumenko, A. Potylitsyn, N. Terunuma, and J. Urakawa, Beam-Size Measurement with Optical Diffraction Radiation at KEK Accelerator Test Facility, Phys. Rev. Lett. 93, 244802 (2004).

[14] B. Bolzon et al., Very high-resolution optical transition radiation imaging system: Comparison between simulation and experiment, Phys. Rev. Accel. Beams 18, 082803 (2015).

[15] T. Lefèvre et al., Non-invasive beam diagnostics with Cherenkov diffraction radiation, in Proceedings of IPAC'18, Vancouver, BC, Canada (JACoW Publishing, Geneva, Switzerland, 2018), p. 2005. 